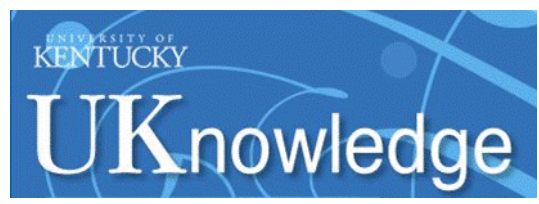

University of Kentucky

UKnowledge

$12-13-2013$

\title{
Atomic Data for S II-Toward Better Diagnostics of Chemical Evolution in High-Redshift Galaxies
}

\author{
Romas Kisielius \\ Vilnius University, Lithuania \\ Varsha P. Kulkarni \\ University of South Carolina \\ Gary J. Ferland \\ University of Kentucky, gary@uky.edu \\ Pavel Bogdanovich \\ Vilnius University, Lithuania \\ Matt L. Lykins \\ University of Kentucky, mllyki3@uky.edu
}

Follow this and additional works at: https://uknowledge.uky.edu/physastron_facpub

Part of the Astrophysics and Astronomy Commons, and the Physics Commons

Right click to open a feedback form in a new tab to let us know how this document benefits you.

\section{Repository Citation}

Kisielius, Romas; Kulkarni, Varsha P.; Ferland, Gary J.; Bogdanovich, Pavel; and Lykins, Matt L., "Atomic Data for S II-Toward Better Diagnostics of Chemical Evolution in High-Redshift Galaxies" (2013). Physics and Astronomy Faculty Publications. 56.

https://uknowledge.uky.edu/physastron_facpub/56

This Article is brought to you for free and open access by the Physics and Astronomy at UKnowledge. It has been accepted for inclusion in Physics and Astronomy Faculty Publications by an authorized administrator of UKnowledge. For more information, please contact UKnowledge@lsv.uky.edu. 


\section{Atomic Data for S II-Toward Better Diagnostics of Chemical Evolution in High- Redshift Galaxies}

Digital Object Identifier (DOI)

https://doi.org/10.1088/0004-637X/780/1/76

Notes/Citation Information

Published in The Astrophysical Journal, v. 780, no. 1, 76, p. 1-11.

(c) 2014. The American Astronomical Society. All rights reserved. Printed in the U.S.A.

The copyright holder has granted permission for posting the article here. 


\title{
ATOMIC DATA FOR S II-TOWARD BETTER DIAGNOSTICS OF CHEMICAL EVOLUTION IN HIGH-REDSHIFT GALAXIES
}

\author{
Romas Kisielius ${ }^{1}$, Varsha P. Kulkarni ${ }^{2}$, Gary J. Ferland ${ }^{3}$, Pavel Bogdanovich ${ }^{1}$, and Matt L. Lykins ${ }^{3}$ \\ ${ }^{1}$ Institute of Theoretical Physics and Astronomy, Vilnius University, A. Goštauto 12, LT-01108, Lithuania \\ ${ }^{2}$ Department of Physics and Astronomy, University of South Carolina, Columbia, SC 29208, USA \\ ${ }^{3}$ Department of Physics and Astronomy, University of Kentucky, Lexington, KY 40506, USA \\ Received 2013 August 24; accepted 2013 November 10; published 2013 December 13
}

\begin{abstract}
Absorption-line spectroscopy is a powerful tool used to estimate element abundances in both the nearby and distant universe. The accuracy of the abundances thus derived is naturally limited by the accuracy of the atomic data assumed for the spectral lines. We have recently started a project to perform new extensive atomic data calculations used for optical/UV spectral lines in the plasma modeling code Cloudy using state of the art quantal calculations. Here, we demonstrate our approach by focussing on S II, an ion used to estimate metallicities for Milky Way interstellar clouds as well as distant damped Lyman-alpha (DLA) and sub-DLA absorber galaxies detected in the spectra of quasars and gamma-ray bursts. We report new extensive calculations of a large number of energy levels of S II, and the line strengths of the resulting radiative transitions. Our calculations are based on the configuration interaction approach within a numerical Hartree-Fock framework, and utilize both non-relativistic and quasirelativistic one-electron radial orbitals. The results of these new atomic calculations are then incorporated into Cloudy and applied to a lab plasma, and a typical DLA, for illustrative purposes. The new results imply relatively modest changes $(\approx 0.04 \mathrm{dex})$ to the metallicities estimated from $\mathrm{S}$ II in past studies. These results will be readily applicable to other studies of S II in the Milky Way and other galaxies.
\end{abstract}

Key words: atomic data - atomic processes - galaxies: abundances - ISM: abundances - quasars: absorption lines

Online-only material: color figures, machine-readable table

\section{INTRODUCTION}

A powerful tool for studying distant galaxies is provided by absorption lines in the spectra of quasars superposed by foreground galaxies along the sightline, which are sampled simply by gas cross section, independent of their brightness. Damped Lyman-alpha (DLAs; neutral hydrogen column densities $N_{\mathrm{H}_{\mathrm{I}}} \geqslant 2 \times 10^{20} \mathrm{~cm}^{-2}$ ) absorbers and sub-DLAs $\left(10^{19} \leqslant N_{\mathrm{HI}_{\mathrm{I}}}<2 \times 10^{20} \mathrm{~cm}^{-2}\right)$ are especially useful for this purpose. These are the primary neutral gas reservoir for star formation at redshifts $0<z<5$ (e.g., Storrie-Lombardi \& Wolfe 2000; Péroux et al. 2005; Prochaska et al. 2005). Over the past decade, DLAs toward gamma-ray burst afterglows have also emerged as a powerful probe of distant galaxies (e.g., Savaglio et al. 2003; Chen et al. 2005; Prochaska et al. 2007; Fynbo et al. 2009).

The elemental compositions of DLAs/sub-DLAs offer highly sensitive tracers of the chemical evolution of galaxies (e.g., Pettini et al. 1997; Kulkarni \& Fall 2002; Prochaska et al. 2003, 2007; Kulkarni et al. 2007; Péroux et al. 2008; Meiring et al. 2009; Cooke et al. 2011; Rafelski et al. 2012; Som et al. 2013). Element abundances in the absorbers are determined from optical/UV atomic resonance lines. Volatile elements such as $\mathrm{N}, \mathrm{O}, \mathrm{P}, \mathrm{S}, \mathrm{Ar}$, and $\mathrm{Zn}$ are not strongly condensed on interstellar dust grains, so their gas-phase abundances can give their total (gas + solid phase) abundances. In practice, for reasons such as wavelength coverage and availability of suitable lines, $\mathrm{S}$ and $\mathrm{Zn}$ have emerged as the most commonly used metallicity indicators used for DLAs. The common ionization stage of S seen in cool interstellar clouds and DLAs is S II. S II has several absorption lines that can be used to obtain reliable column densities despite their usual presence in the Ly $\alpha$ forest.

The accuracy of the element abundances and physical properties inferred from them depends crucially on the quality of the atomic data used. By far, the most commonly used atomic data reference for DLA spectral analysis is Morton (2003; see, e.g., Battisti et al. 2012; Rafelski et al. 2012; Kulkarni et al. 2012; Guimaraes 2012; Jorgenson 2013; Som et al. 2013). For some important transitions and ions, the oscillator strengths listed in Morton (2003) have relatively large uncertainties (as listed on the NIST Atomic Spectra Database; Kramida et al. 2013), while for other transitions, oscillator strengths are not available at all. In some cases, even more recent values obtained since Morton (2003) have low accuracy grades listed in the NIST database. Limitations in atomic data can compromise our ability to read the messages received from high-redshift galaxies. To produce new, reliable atomic data for commonly used astrophysical ions, we have recently started a collaborative study that brings together atomic physics, plasma simulations, and observational spectroscopy. Our goals are to assess the quality of the existing atomic data, to improve the accuracy of the data that were designated low accuracies, to incorporate them into our widely used plasma simulation code Cloudy, and to apply it to existing/new observations of high-redshift galaxies such as DLAs/sub-DLAs. Here, we provide an early illustration of our approach by focussing on $\mathrm{S}_{\text {II, }}$ an ion of great importance for DLA element abundance studies.

Since it is a volatile element, $\mathrm{S}$ does not condense easily on interstellar dust grains. In the Milky Way, S shows a depletion of $<0.1 \mathrm{dex}$ in cool as well as warm interstellar clouds (e.g., Savage \& Sembach 1996; but see also Jenkins 2009 for the suggestion that the true depletion of S could be larger in the presence of ionization effects). The relatively low depletion makes $\mathrm{S}$ ideal for estimating metallicity from gas-phase abundance measurements. Moreover, $\mathrm{S}$ is a fairly abundant element, so its absorption lines are easily detectable (more easily detectable than the lines of $\mathrm{Zn}$, another nearly undepleted element). Especially important among the $\mathrm{S}$ ions 
is $\mathrm{S}$ II, which is the dominant ion in DLAs. S II has a number of absorption lines, especially a triplet at $\lambda \lambda 1250.6,1253.8$, 1259.5 , which are strong enough to be detectable. The weakest of these lines can be relatively unsaturated, giving reasonably accurate column densities (although the stronger lines can be saturated). Studies of DLAs, especially at $z \gtrsim 2$, often use these three $\mathrm{S}$ II lines to derive $[\mathrm{S} / \mathrm{H}]$ and adopt that as the gasphase metallicity. Studies of the Milky Way interstellar gas have also often adopted $\mathrm{S}$ as a metallicity indicator. For all of these calculations, it is naturally important to use accurate atomic data for S II.

Morton (2003) lists the oscillator strengths for the above mentioned three S II lines to be 0.00543, 0.0109, and 0.0166, respectively, but the uncertainties in these values are classified as "C" grade, i.e., at a level of about $25 \%$ as per the NIST database. Thus, the uncertainties in the metallicity introduced by the uncertainties in the oscillator strength $(\sim 0.1 \mathrm{dex})$ are far larger than those often quoted from the measurement uncertainties in high-resolution data (typically $\lesssim 0.05 \mathrm{dex}$ ). S II also has absorption lines at $906.9,910.5$, and $912.7 \AA$, but they are too close to the hydrogen Lyman edge and much stronger, and hence likely saturated. Other S II lines at 943.0, 947.0, 1021.3, and 1021.5 $\AA$ are also listed in Morton (2003), but without oscillator strength estimates.

With the intent of assessing the accuracy of the atomic data, we undertook new calculations of the oscillator strengths for all $\mathrm{S}$ II electric dipole, magnetic dipole, and electric quadrupole transitions. Section 2 describes these new calculations and compares them to previous estimates. Section 3 describes the incorporation of these calculations into Cloudy, and Section 4 discusses the implications for DLA abundance studies.

\section{CALCULATIONS OF NEW ATOMIC DATA}

A broad study of energy levels, oscillator strengths, and transition probabilities for the levels of some low configurations of S II was performed by Irimia \& Froese Fischer (2005). The authors used a multiconfiguration Hartree-Fock (MCHF) method with relativistic effects included in the Breit-Pauli (BP) approximation in their study. They have determined all possible allowed E1 and many forbidden (E2, M1) transitions for the states under consideration and determined level lifetimes and splittings. As a first step in these calculations, ab initio wavefunctions were obtained, and then the diagonal energies of $L S$ blocks were adjusted in order to get better agreement for the energies of the $L S$ terms with the observed values. We refer to the results of these calculations as $\mathrm{MCHF}_{05}$.

A subsequent study by Froese Fischer et al. (2006) considered energy levels, lifetimes, and transition probabilities for several sequences, including the $\mathrm{S}_{\mathrm{II}}$ ion as a member of the $P$-like sequence. These authors used several theoretical methods, such as non-orthogonal spline configuration interaction (CI), MCHF, and multiconfiguration Dirac-Hartree-Fock. Transitions between the computed levels were reported for allowed E1 and some forbidden (M1, M2, E2, E3) transitions. The MCHF wavefunction expansion adopted in this work was very similar to that of Irimia \& Froese Fischer (2005), but there were no term corrections included in the Hamiltonian matrix. In the comparisons given below, we will use the results of the Froese Fischer et al. (2006) calculations as consistent CI ab initio calculation data and refer to them as $\mathrm{MCHF}_{06}$.

Recently, Tayal \& Zatsarinny (2010) reported new calculations for transition probabilities and electron impact collision strengths for the astrophysically important lines in $\mathrm{S}$ II. The
MCHF method with term-dependent non-orthogonal orbitals was employed for accurate representation of the target wavefunctions. Relativistic corrections were included in the BP approximation. Their close-coupling expansion included 70 bound levels of S II covering all possible terms of the ground $3 s^{2} 3 p^{3}$ configuration and singly excited $3 s 3 p^{4}, 3 s^{2} 3 p^{2} 3 d, 3 s^{2} 3 p^{2} 4 s$, and $3 s^{2} 3 p^{2} 4 p$ configurations. This approach made it possible to achieve a more accurate description of both energy levels and oscillator strengths with a relatively small CI expansion compared to that of more traditional methods with an orthogonal set of one-electron orbitals where large CI expansions are necessary. According to Tayal \& Zatsarinny (2010), the accuracy of their calculations is comparable with the accuracy of the BP MCHF calculations by Irimia \& Froese Fischer (2005) discussed earlier. Hereafter, we refer to the calculations of Tayal \& Zatsarinny (2010) as $\mathrm{MCHF}_{\mathrm{TD}}$.

A systematic study of forbidden M1 and E2 transitions for P I, S II, Cl III, and Ar IV was reported by Fritzsche et al. (1999). They applied multiconfiguration Dirac-Fock wavefunctions of different sizes to check for the convergence of results. These authors concluded that the convergence and reasonable agreement of their calculations with previously determined results could be achieved only after a large number of valence- and core-excited configurations were included in their multiconfiguration wavefunction expansion.

The above mentioned works are the most systematic and complex theoretical studies of allowed and forbidden transitions in S II so far. They provide more reliable line data compared to the earlier relativistic results of Mendoza \& Zeippen (1982) and Keenan et al. (1993). A recent compilation by Podobedova et al. (2009) tabulated more than 6000 allowed and forbidden lines of S I to S XV. This study provides a critical evaluation of recent theoretical values for transition rates, and also includes energy level values that are primarily experimental, taken from the NIST compilation by Kramida et al. (2013). Specifically for S II, Podobedova et al. (2009) list transition probabilities for allowed E1 transitions and forbidden M1 and E2 transitions. In this compilation, the estimated uncertainties of theoretical values in many E1 transitions exceed $25 \%$ while M1 and E2 transitions are given better accuracy.

In this work, we employ two different approximations for the calculation of $\mathrm{S}_{\text {II }}$ transition rates. In the first approximation, an MCHF method is adopted where relativistic corrections are included in the BP approximation. It resembles the method used in Froese Fischer et al. (2006), but there are several significant differences. We adopt transformed radial orbitals (TROs; Bogdanovich 2004, 2005) in order to efficiently include electron correlation corrections caused by excited configurations with higher principal quantum numbers $n>4$. In this work, the transformed one-electron radial orbitals $P_{\mathrm{TRO}}(n l \mid r)$ have two variational parameters, an integer and even $k$ and a positive $B$ :

$$
\begin{aligned}
P_{\mathrm{TRO}}(n l \mid r)= & N\left(r^{l-l_{0}+k} \exp (-B r) P\left(n_{0} l_{0} \mid r\right)\right. \\
& -\sum_{n^{\prime}<n} P\left(n^{\prime} l \mid r\right) \int_{0}^{\infty} P\left(n^{\prime} l \mid r^{\prime}\right) r^{\prime\left(l-l_{0}+k\right)} \\
& \left.\times \exp \left(-B r^{\prime}\right) P\left(n_{0} l_{0} \mid r^{\prime}\right) d r^{\prime}\right) .
\end{aligned}
$$

Here, the factor $N$ ensures the normalization of the determined TROs, the first term in the parenthesis performs the transformation of RO based on the one-electron radial orbital $P\left(n_{0} l_{0} \mid r\right)$ from the set of investigated configurations, and the second term ensures their orthogonality. The parameters $k$ and $B$ are chosen to gain the maximum of the energy correlation correction. In 
the current calculation, TROs were determined for the configurations with the outer electron that have a principal quantum number $5 \leqslant n \leqslant 7$ and all allowed values of the orbital quantum number $l$.

The second rather significant difference is in the selection of the configurations included in the CI wave function expansion. Instead of simply including higher- $n$ excited configurations, we follow the procedure described by Bogdanovich \& Karpuškienè (2001) and remove those configurations (admixed configurations) within the CI wavefunction expansion of the investigated configuration (adjusted configuration) that have a mean weight $\bar{W}_{\text {PT }}<1 \times 10^{-8}$.

The parameter $\bar{W}_{\mathrm{PT}}$ is determined in the second order of perturbation theory $(\mathrm{PT})$ :

$$
\begin{aligned}
& \bar{W}_{\mathrm{PT}}\left(K_{0}, K^{\prime}\right) \\
& =\frac{\sum_{T L S T^{\prime}}(2 L+1)(2 S+1)\left\langle K_{0} T L S\|H\| K^{\prime} T^{\prime} L S\right\rangle^{2}}{g\left(K_{0}\right)\left(\bar{E}\left(K^{\prime}\right)-\bar{E}\left(K_{0}\right)\right)^{2}},
\end{aligned}
$$

where $\left\langle K_{0} T L S\|H\| K^{\prime} T^{\prime} L S\right\rangle$ is a Hamiltonian matrix element for the interaction between the adjusted $K_{0}$ and admixed $K^{\prime}$ configuration $L S$ terms, $g$ is the statistical weight of the configuration $K_{0}$, and $\bar{E}$ are the averaged energies of the configurations. This method, paired with the methods from Bogdanovich et al. (2002), makes it possible to significantly reduce the size of the Hamiltonian matrices.

In the CI approximation, our methods allow for the use of two kinds of radial orbitals describing the electrons of adjusted configurations that are applied for the transformations (1). In the case of $\mathrm{S}_{\mathrm{II}}$, we have electrons with a principal quantum number of $n \leqslant 4$. Traditionally, the solutions of the standard Hartree-Fock equations are utilized for this purpose (see, e.g., Bogdanovich et al. 2003; Jonauskas et al. 2005, 2006; Karpuškienė \& Bogdanovich 2009). We use the notation $\mathrm{CI}_{\mathrm{HF}+\mathrm{TRO}}$ to denote the results obtained for this approximation. Relativistic corrections are included in the BP approximation, as in the MCHF calculations.

In order to partially account for relativistic corrections at the stage when the one-electron radial orbitals are determined, we developed a new method that solves the quasirelativistic (QR) Hartree-Fock equations. The QR radial orbitals, obtained after solving the $\mathrm{QR}$ equations, are applied to determine the one-electron wavefunctions of admixed configurations and also to calculate the TROs given by Equation (1). The consequent inclusion of correlation effects is achieved using the same method as in the case of the aforementioned non-relativistic Hartree-Fock radial orbitals. To determine the energy levels, the $\mathrm{BP}$ approximation is applied as in the $\mathrm{CI}_{\mathrm{HF}+\mathrm{TRO}}$ calculations. We use the $\mathrm{CI}_{\mathrm{QR}+\mathrm{TRO}}$ notation for this method. Furthermore, we must mention that our QR method differs significantly from the more traditional QR method of Cowan (1981). A more detailed description of the applied QR approximation can be found elsewhere (see, e.g., Bogdanovich \& Rancova 2006, 2007, 2008; Bogdanovich \& Kisielius 2012, 2013).

\section{ACCURACY OF ATOMIC DATA SETS}

One of the main tasks of the present paper is to assess the accuracy of the spectroscopic data used in modeling the S II emission or absorption spectra. In order to do that, we compare our results, both in the $\mathrm{CI}_{\mathrm{HF}+\mathrm{TRO}}$ and $\mathrm{CI}_{\mathrm{QR}+\mathrm{TRO}}$ approximations, with those from the Froese Fischer et al. (2006) and Tayal \& Zatsarinny (2010) calculations.
Table 1

\begin{tabular}{|c|c|c|c|c|c|c|c|}
\hline$N$ & State & $J$ & $\mathrm{CI}_{\mathrm{HF}+\mathrm{TRO}}$ & $\mathrm{CI}_{\mathrm{QR}+\mathrm{TRO}}$ & Exp & $\mathrm{MCHF}_{06}$ & $\mathrm{MCHF}_{\mathrm{TD}}$ \\
\hline 1 & $3 p^{34} S$ & 1.5 & 0 & 0 & 0 & 0 & \\
\hline 2 & $3 p^{32} D$ & 1.5 & 15292 & 15320 & 14853 & 15282 & 14880 \\
\hline 3 & $3 p^{32} D$ & 2.5 & 15307 & & & & 14905 \\
\hline 4 & $3 p^{32} P$ & 0.5 & 25407 & 25549 & 24525 & 24817 & 24632 \\
\hline 5 & $3 p^{32} P$ & 1.5 & 25431 & 25574 & 24572 & 24848 & 24656 \\
\hline 6 & $3 s 3 p^{4}{ }^{4} P$ & 2.5 & 77787 & 78926 & 9395 & 78468 & 9405 \\
\hline & $3 s 3 p^{44} P$ & 1.5 & 78106 & 79249 & 79757 & 78763 & 79704 \\
\hline & $3 s 3 p^{44} P$ & 0.5 & 78290 & 79434 & 79963 & 78929 & 79873 \\
\hline & $3 s 3 p^{42} D$ & 2.5 & 97281 & 98322 & 7919 & 97500 & 97875 \\
\hline 10 & $3 s 3 p^{42} D$ & 1.5 & 97284 & 98324 & 97891 & 97481 & 97899 \\
\hline & $3 p^{2} 3 d^{2} P$ & 1.5 & 105241 & 105702 & 105599 & 105444 & 105530 \\
\hline 2 & $3 p^{2} 3 d^{2} P$ & 0.5 & & & & & \\
\hline 3 & $3 p^{2} 4 s^{4} P$ & 0.5 & 109399 & 4 & 61 & & 9635 \\
\hline 4 & $3 p^{2} 4 s^{4} P$ & 1.5 & 109600 & & & & 9877 \\
\hline 5 & $3 p^{2} 4 s^{4} P$ & 2.5 & 109927 & 9880 & 10269 & 09570 & 110264 \\
\hline 6 & $3 p^{2} 3 d^{4} F$ & 1.5 & 10068 & 0275 & & 10174 & 110216 \\
\hline 7 & $3 p^{2} 3 d^{4} F$ & 2.5 & 0179 & & & 297 & 0337 \\
\hline 8 & $3 p^{2} 3 d^{4} F$ & 3.5 & 110336 & & & & 110514 \\
\hline & $3 p^{2} 3 d^{4} F$ & 4.5 & 110543 & & & & 110748 \\
\hline & $3 p^{2} 4 s^{2} P$ & 0.5 & 3060 & & & 487 & 3055 \\
\hline 1 & $3 p^{2} 4 s^{2} P$ & 1.5 & 3449 & 13422 & 13462 & 952 & 13514 \\
\hline 22 & $3 p^{2} 3 d^{4} D$ & 0.5 & & & & & 4144 \\
\hline 23 & $3 p^{2} 3 d^{4} D$ & 1.5 & & & & & 4176 \\
\hline 24 & $3 p^{2} 3 d^{4} D$ & 2.5 & & & & & \\
\hline 5 & $3 p^{2} 3 d^{4} D$ & 3.5 & & & & & \\
\hline 6 & $3 p^{2} 3 d^{2} F$ & 2.5 & & & & & 853 \\
\hline & $3 p^{2} 3 d^{2} F$ & 3.5 & & & & & 281 \\
\hline 8 & $3 s 3 p^{42} S$ & 0.5 & & & & & \\
\hline 9 & $3 p^{2} 4 s^{2} D$ & 1.5 & & & & & 121499 \\
\hline 30 & $3 p^{2} 4 s^{2} D$ & 2.5 & 1658 & 1614 & & & 1499 \\
\hline & $3 p^{2} 3 d^{2} G$ & 3.5 & 127320 & 27489 & 27 & 127771 & 127161 \\
\hline & $3 p^{2} 3 d^{2} G$ & 4.5 & 127332 & 127501 & & 127773 & 127161 \\
\hline 3 & $3 p^{2} 3 d^{4} P$ & 2.5 & & & & & 130775 \\
\hline 34 & $3 p^{2} 3 d^{4} P$ & 1.5 & & & & & 130960 \\
\hline 5 & $3 p^{2} 3 d^{4} P$ & 0.5 & & & 49 & & 131073 \\
\hline 6 & $3 p^{2} 3 d^{2} D$ & 1.5 & & & & & 469 \\
\hline 7 & $3 p^{2} 3 d^{2} D$ & 2.5 & & & & 46 & 33864 \\
\hline 8 & $3 p^{2} 4 s^{2} S$ & 0.5 & 6559 & 36753 & 6329 & 136026 & 136315 \\
\hline 9 & $3 p^{2} 3 d^{2} P$ & 0.5 & 0742 & 40939 & 9845 & 140485 & 139881 \\
\hline & $3 p^{2} 3 d^{2} P$ & 1.5 & 140826 & 41021 & & 140667 & 140034 \\
\hline 41 & $3 p^{2} 3 d^{2} F$ & 3.5 & 141879 & 142499 & 38527 & 139950 & 138639 \\
\hline & $3 p^{2} 3 d^{2} F$ & 2.5 & 141896 & 142516 & 38509 & 139956 & 138614 \\
\hline & $3 p^{2} 3 d^{2} D$ & 2.5 & 145843 & 146418 & 44009 & 144982 & 144308 \\
\hline & $3 p^{2} 3 d^{2} D$ & 1.5 & & & & & 144422 \\
\hline & $3 s 3 p^{42} P$ & 1.5 & 152020 & 153750 & 145506 & 145933 & 145631 \\
\hline 46 & $3 s 3 p^{42} P$ & 0.5 & & & & & 145946 \\
\hline 47 & $3 p^{2} 3 d^{2} D$ & 2.5 & & & & & 149067 \\
\hline 48 & $3 p^{2} 3 d^{2} D$ & 1.5 & 152491 & 153257 & 148901 & 150614 & 149075 \\
\hline 40 & $3 p^{2} 3 d^{2} S$ & 0.5 & 154834 & 155791 & 151652 & 153365 & 151745 \\
\hline \multicolumn{3}{|c|}{ USI } & 1710 & 2071 & & 698 & 94 \\
\hline
\end{tabular}

Comparison of Calculated S II Energy Levels with Experimental Data

Notes. $\mathrm{CI}_{\mathrm{HF}+\mathrm{TRO}}$-our $\mathrm{HF}$ data; $\mathrm{CI}_{\mathrm{QR}+\mathrm{TRO}}$ - our quasirelativistic data; Exp-experimental values; $\mathrm{MCHF}_{06}$ - data from Froese Fischer et al. (2006); $\mathrm{MCHF}_{\mathrm{TD}}$ —data from Tayal \& Zatsarinny (2010).

\subsection{Energy Levels and Wavelengths}

Table 1 presents the energy levels of $S_{\text {II. The results of }}$ our calculations obtained with the two methods are compared with the experimental data and with the values calculated by Froese Fischer et al. (2006) and Tayal \& Zatsarinny (2010). One can see from the mean-square deviations (MSD) provided at the bottom of Table 1 that the data of Tayal \& Zatsarinny (2010) agree with the experimental values better than with 


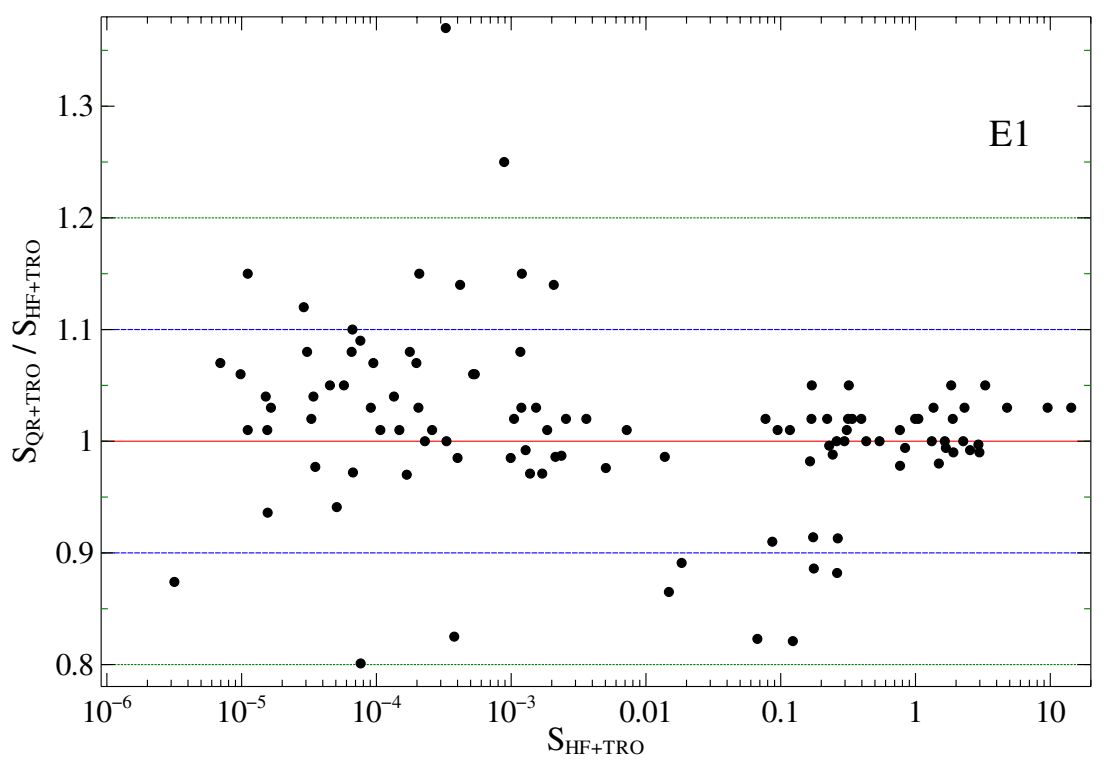

Figure 1. Comparison of the $\mathrm{E} 1$ transition line strengths $S$ determined in the $\mathrm{CI}_{\mathrm{HF}+\mathrm{TRO}}$ and $\mathrm{CI}_{\mathrm{QR}+\mathrm{TRO}}$ approaches for $\mathrm{S}$ II.

(A color version of this figure is available in the online journal.)

both sets of our data or those of Froese Fischer et al. (2006), although the differences among the calculated values are rather small. The better accuracy of the Tayal \& Zatsarinny (2010) data can be explained by the fact that those authors used the term-dependent non-orthogonal radial orbitals to calculate energy levels and radiative transition parameters. Such an approximation determines the eigenvalues for each $L S$ term separately instead of optimizing a complete set of terms.

Nevertheless, some deviations in energy level values (and consequently, in transition wavelength values) are not significant, and they do not exceed 3\%. Moreover, these discrepancies can be overcome by using the experimental wavelengths, which are well-known for the most important S II lines, for the line identification or by using the experimental energy differences to determine the "corrected" oscillator strengths or radiative transition probabilities (see Verner et al. 1996).

\subsection{E1 Lines}

We have determined radiative transition parameters for the lines arising from the transitions among the levels of $3 s^{2} 3 p^{3}$, $3 s 3 p^{4}, 3 p^{2} 3 d$, and $3 p^{2} 4 s$ configurations of $\mathrm{S}$ II. The electric dipole, electric qudrupole, and magnetic dipole transitions were considered. The main target of the present work is not only to determine high-accuracy radiative transition data but also to evaluate the accuracy of the calculations and their suitability for use in the plasma modeling code Cloudy.

One of the ways to assess the accuracy of our results is to compare them with other available data. For this purpose, we compare the E1 transition line strengths $S$. Transition line strengths $S$ are chosen because they do not depend directly on the transition energy difference, unlike the oscillator strengths $f$ or transition probabilities $A$. As was mentioned previously, the accuracy of $f$ values and $A$ values can be increased by using theoretically calculated $S$ values and the experimental transition energy or the wavelength $\lambda$.

Figure 1 compares our calculated results determined using two different approximations. The $\mathrm{CI}_{\mathrm{HF}+\mathrm{TRO}}$ approach utilizes non-relativistic radial orbitals while the $\mathrm{CI}_{\mathrm{QR}+\mathrm{TRO}}$ approach employs QR radial orbitals. These two approaches adopt the same CI method, involving the TROs, to deal with the correlation effects. As one can see, the agreement between the results is rather nice, especially for the strongest lines. Within two orders of magnitude, the discrepancies do not exceed $10 \%$, except for three lines. Within four orders of magnitude, the most discrepancies are within $10 \%$, but there are five more lines with the discrepancies ranging from $10 \%$ to $20 \%$. Extending the comparison to six orders of magnitude, the situation does not change substantially, and most lines agree within $10 \%$. Nevertheless, there are some lines where discrepancies exceed $20 \%$ or even $30 \%$.

A comparison of our $\mathrm{CI}_{\mathrm{HF}+\mathrm{TRO}}$ calculation results with the data from Froese Fischer et al. (2006) is given in Figure 2. As can be seen, the agreement between these data is worse in Figure 2 compared to Figure 1. This is caused by the use of different CI expansion bases in our calculations and those of Froese Fischer et al. (2006). In general, the discrepancies for most of the strong lines are within $20 \%$, except for the few lines with discrepancies larger than that, when transition line strengths within three orders of magnitude are considered. One can see some larger deviations, exceeding 30\% in this comparison, but there are few such lines. For weaker lines, there is a large number of lines with discrepancies exceeding $30 \%$.

In Figure 3, a similar comparison of our $\mathrm{CI}_{\mathrm{HF}+\mathrm{TRO}}$ results with the data from Tayal \& Zatsarinny (2010) is presented. Here again, the discrepancies do not exceed $30 \%$ and in most cases are below $20 \%$ for the line strength in the range of four orders of magnitude. There are just five lines with discrepancies exceeding $25 \%$. When weaker lines are considered, the agreement for data resulting from different approximations is worse, and in some cases the discrepancies can reach $50 \%$ or even more.

We conclude that our two sets of calculations for the radiative transition data agree very well between themselves for the strongest transitions, within six orders of magnitude of the largest line strengths. This proves that relativistic corrections are adequately included in our $\mathrm{CI}_{\mathrm{HF}+\mathrm{TRO}}$ approximation. For the weakest lines where transitions purely depend on CI effects, this close agreement breaks down even if the CI basis remains the same. A comparison with the data from Froese Fischer et al. (2006) and Tayal \& Zatsarinny (2010) demonstrates that for 


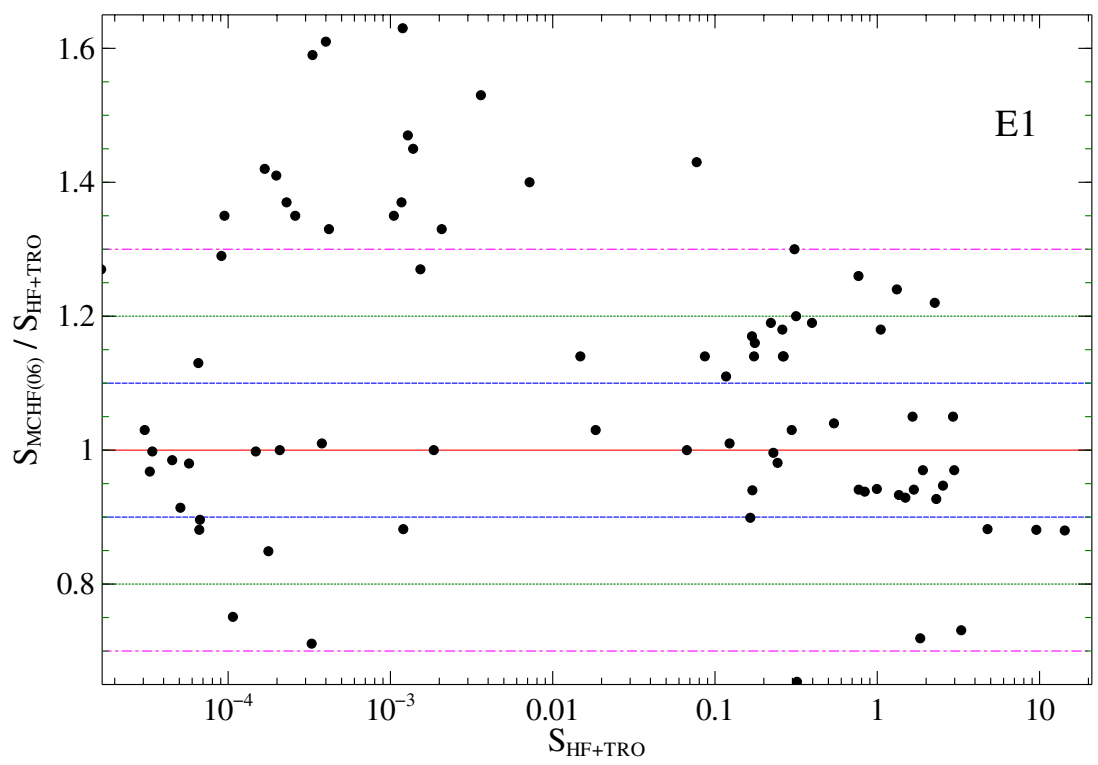

Figure 2. Comparison of the $\mathrm{E} 1$ transition line strengths $S$ determined in the $\mathrm{CI}_{\mathrm{HF}+\mathrm{TRO}}$ and $\mathrm{MCHF}_{06}$ (Froese Fischer et al. 2006) approaches for $\mathrm{S}$ II. (A color version of this figure is available in the online journal.)

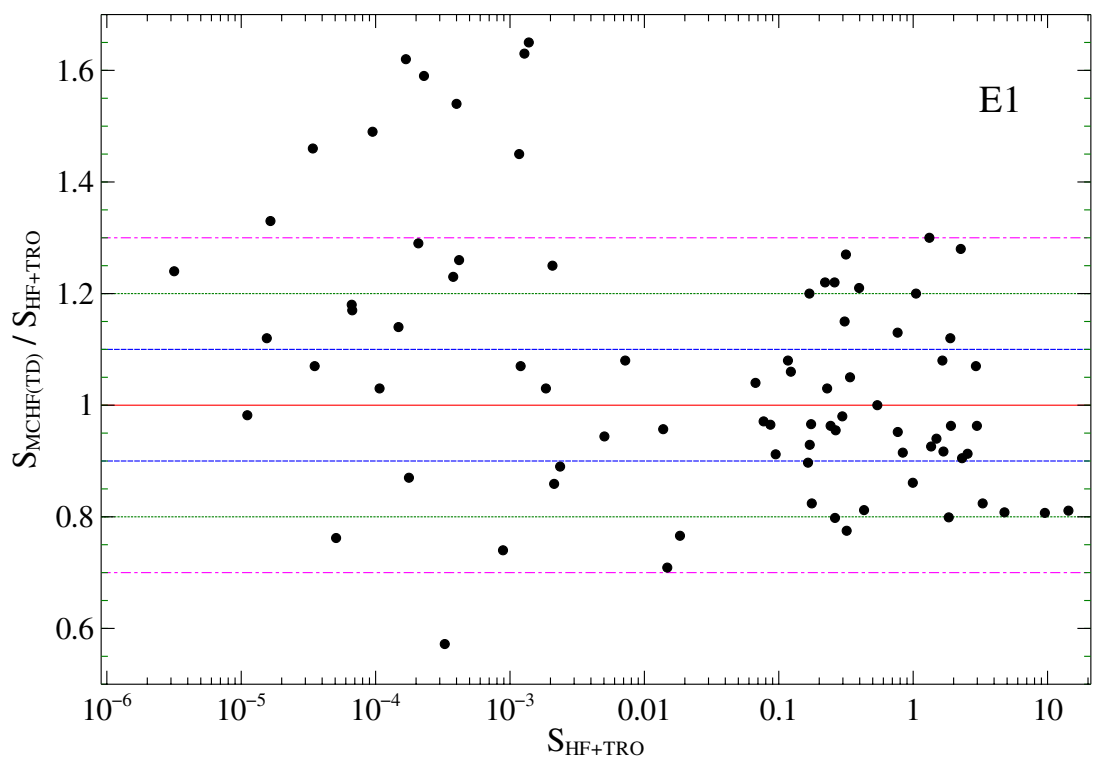

Figure 3. Comparison of the $\mathrm{E} 1$ transition line strengths $S$ determined in the $\mathrm{CI}_{\mathrm{HF}+\mathrm{TRO}}$ and $\mathrm{MCHF}_{\mathrm{TD}}$ (Tayal \& Zatsarinny 2010) approaches for $\mathrm{S}$ II.

(A color version of this figure is available in the online journal.)

the strongest lines, deviations are within $20 \%-30 \%$ for the line strengths varying by four orders of magnitude from the strongest. The agreement for many weaker lines is worse and exceeds $30 \%$, as the aforementioned studies apply different CI bases compared to our calculations.

The particular $\mathrm{S}_{\text {II }}$ lines listed in Table 2 were chosen because they are all of the lines from S II listed by Morton (2003), which is the commonly used reference in observational spectroscopy of quasar absorption systems and the Galactic interstellar medium (ISM). The most commonly used lines are the triplet at $1250.578,1253.805,1259.518 \AA$, as they have determinations of oscillator strengths and are relatively easy to observe, being longward of the H I Ly $\alpha$ transition, and often outside the red wing of the Ly $\alpha$ transition. The doublets at or below $912 \AA$ are difficult to observe due to their proximity to the H I Lyman limit. In the case of DLA/sub-DLAs (and in general the Lyman-limit systems), absorption near the H I Lyman limit renders the quasar flux to be nearly zero in this wavelength region. Nevertheless, we list them in Table 2 for the sake of completeness. The doublets at $1021.254,1021.539 \AA$ and at 943.003, $946.978 \AA$ can in principle be observed with more ease, but have no measurements of oscillator strengths listed in Morton (2003). From our calculations for these lines, as well as those from other works presented in Table 2, it is clear that observing these lines will be far more challenging. However, the strongest of these lines (at $1021.539 \AA$ ) may be possible to detect in the strongest absorbers. For example, in a solar-metallicity absorber with $\log N_{\mathrm{HI}}=22.0$, the S II $\lambda 1021.254$ line would be expected to have a rest-frame equivalent width of $\approx 12 \mathrm{~m} \AA$.

In the last column of Table 2 , we present the $g f$ values for these lines. These values are derived from the line strengths $S$ calculated in the $\mathrm{CI}_{\mathrm{HF}+\mathrm{TRO}}$ approximation with the 
Table 2

Comparison of Calculated Transition Rates $A\left(s^{-1}\right)$ for Some E1 Transitions to the Ground State $3 p^{3}{ }^{4} S_{3 / 2}$

\begin{tabular}{|c|c|c|c|c|c|c|c|c|}
\hline Excited State & $\begin{array}{c}\lambda_{\operatorname{Exp}} \\
(\AA)\end{array}$ & $a g$ & $\mathrm{CI}_{\mathrm{HF}+\mathrm{TRO}}$ & $\mathrm{CI}_{\mathrm{QR}+\mathrm{TRO}}$ & $\mathrm{MCHF}_{\mathrm{TD}}$ & $\mathrm{MCHF}_{06}$ & $\mathrm{MCHF}_{05}$ & $g f^{\text {corr }}$ \\
\hline $3 s 3 p^{4}{ }^{4} P_{5 / 2}$ & 1259.518 & $D+$ & $4.48 \mathrm{E}+7$ & $4.02 \mathrm{E}+7$ & $4.27 \mathrm{E}+7$ & $4.92 \mathrm{E}+7$ & $5.10 \mathrm{E}+7$ & $6.27 \mathrm{E}-2$ \\
\hline $3 s 3 p^{4}{ }^{4} P_{3 / 2}$ & 1253.805 & $D+$ & $4.48 \mathrm{E}+7$ & $4.00 \mathrm{E}+7$ & $4.30 \mathrm{E}+7$ & $4.93 \mathrm{E}+7$ & $5.10 \mathrm{E}+7$ & $4.14 \mathrm{E}-2$ \\
\hline $3 s 3 p^{4}{ }^{4} P_{1 / 2}$ & 1250.578 & $D$ & $4.48 \mathrm{E}+7$ & $4.00 \mathrm{E}+7$ & $4.32 \mathrm{E}+7$ & $4.94 \mathrm{E}+7$ & $5.11 \mathrm{E}+7$ & $2.06 \mathrm{E}-2$ \\
\hline $3 s 3 p^{4}{ }^{2} D_{5 / 2}$ & 1021.254 & $n g$ & $3.41 \mathrm{E}+4$ & $3.47 \mathrm{E}+4$ & $3.50 \mathrm{E}+4$ & $2.51 \mathrm{E}+4$ & $2.61 \mathrm{E}+4$ & $3.17 \mathrm{E}-5$ \\
\hline $3 s 3 p^{4}{ }^{2} D_{3 / 2}$ & 1021.539 & $n g$ & $5.28 \mathrm{E}+3$ & $5.40 \mathrm{E}+3$ & $5.17 \mathrm{E}+3$ & $3.54 \mathrm{E}+3$ & $3.67 \mathrm{E}+3$ & $3.28 \mathrm{E}-6$ \\
\hline $3 p^{2}\left({ }^{3} P\right) 3 d^{2} P_{3 / 2}$ & 946.978 & $u l$ & $2.42 \mathrm{E}+2$ & $1.49 \mathrm{E}+3$ & $4.24 \mathrm{E}+3$ & $2.68 \mathrm{E}+3$ & $2.10 \mathrm{E}+3$ & $8.02 \mathrm{E}-7$ \\
\hline $3 p^{2}\left({ }^{3} P\right) 3 d^{2} P_{1 / 2}$ & 943.003 & $u l$ & $9.87 \mathrm{E}+1$ & $1.08 \mathrm{E}+3$ & $9.45 \mathrm{E}+2$ & $1.20 \mathrm{E}+3$ & $7.65 \mathrm{E}+2$ & $2.88 \mathrm{E}-7$ \\
\hline $3 p^{2}\left({ }^{3} P\right) 4 s{ }^{4} P_{1 / 2}$ & 912.735 & $D+$ & $1.12 \mathrm{E}+9$ & $1.10 \mathrm{E}+9$ & $1.02 \mathrm{E}+9$ & $1.03 E+9$ & $1.05 \mathrm{E}+9$ & $2.78 \mathrm{E}-1$ \\
\hline $3 p^{2}\left({ }^{3} P\right) 4 s{ }^{4} P_{3 / 2}$ & 910.484 & $C$ & $1.13 \mathrm{E}+9$ & $1.11 \mathrm{E}+9$ & $1.03 \mathrm{E}+9$ & $1.04 \mathrm{E}+9$ & $1.06 \mathrm{E}+9$ & $5.60 \mathrm{E}-1$ \\
\hline $3 p^{2}\left({ }^{3} P\right) 4 s^{4} P_{5 / 2}$ & 906.885 & $C$ & $1.15 \mathrm{E}+9$ & $1.13 \mathrm{E}+9$ & $1.05 \mathrm{E}+9$ & $1.07 \mathrm{E}+9$ & $1.08 \mathrm{E}+9$ & $8.49 \mathrm{E}-1$ \\
\hline
\end{tabular}

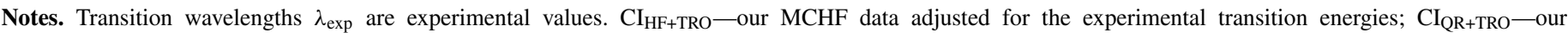

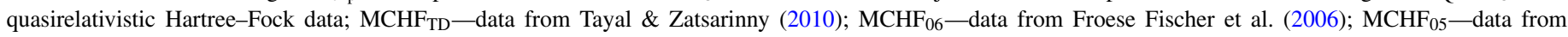

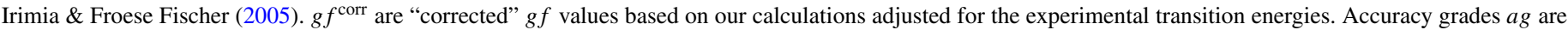

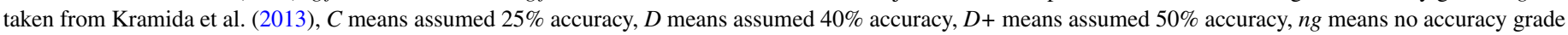
is given, $u l$ means lines are not listed in Kramida et al. (2013).

experimentally adjusted transition energies. We give oscillator strengths as the product of $g f$ due to its symmetry. We note that the emission $f_{u, l}$ and absorption $f_{l, u}$ values are related by $g_{u} f_{u, l}=-g_{l} f_{l, u}$. The lines originating from the excited $3 p^{2}\left({ }^{3} P\right) 3 d^{2} P_{3 / 2}$ and $3 p^{2}\left({ }^{3} P\right) 3 d^{2} P_{1 / 2}$ levels (at 943.003, $946.978 \AA$ ) are very weak. For them, we find the largest discrepancies when different data sets are compared. Unfortunately, our $\mathrm{CI}_{\mathrm{HF}+\mathrm{TRO}}$ results do not agree very well with other data, therefore $g f^{\text {corr }}$ values for these two lines were derived from our $\mathrm{CI}_{\mathrm{QR}+\mathrm{TRO}}$ calculations, which are in much better agreement with the data from Tayal \& Zatsarinny (2010) and Froese Fischer et al. (2006).

\subsection{E2 and M1 Lines}

The line strengths $S$ of E2 transitions from our two sets of calculations are compared in Figure 4. As can be seen, the deviations do not exceed $20 \%$, except for a few weaker lines. However, even for these lines, the deviations are smaller than $30 \%$. Moreover, the deviations for most of the lines are $<10 \%$. So here again, our two calculation methods, $\mathrm{CI}_{\mathrm{HF}+\mathrm{TRO}}$ and $\mathrm{CI}_{\mathrm{QR}+\mathrm{TRO}}$, produce very similar results.

A comparison of our $\mathrm{CI}_{\mathrm{HF}+\mathrm{TRO}}$ results with the data from Froese Fischer et al. (2006) is shown in Figure 5. It is evident that the scatter in the ratio $S_{\mathrm{MCHF}_{06}} / S_{\mathrm{CI}_{\mathrm{HF}+\mathrm{TRO}}}$ is significantly larger compared to the scatter in the $S_{\mathrm{CI}_{\mathrm{QR}+\mathrm{TRO}}} / S_{\mathrm{CI}_{\mathrm{HF}+\mathrm{TRO}}}$ ratio. Nevertheless, the lines within two orders of magnitude of the strongest have deviations smaller than $20 \%$, while most of the remaining weaker lines have deviations smaller than $30 \%$. We note that there are some rather strong lines $(0.1 \leqslant S \leqslant 1)$ that have deviations $>30 \%$ or even $>40 \%$. However, the total number of lines with deviations larger than $30 \%$ is around 20 .

Figure 6 compares our calculated line strengths for the M1 transitions. As can be seen, the agreement is very good. For almost all of the lines, the two values are within $10 \%$ of one another, and only three rather weak lines have deviations worse than 20\%. A similar comparison with the data from Froese Fischer et al. (2006) is given in Figure 7. Unfortunately, in this case, the deviations are much larger, $>20 \%$ or even $30 \%$ for a large fraction of the lines. Since the radiative M1 transition operator does not depend on the variable $r$, the main cause for the large deviations is that different $\mathrm{CI}$ expansion bases are used in our calculation and in the calculation by Froese Fischer et al. (2006).
Table 3

Transition line Strengths $S$ (in a.u.) for S II Determined in the $\mathrm{CI}_{\mathrm{HF}+\mathrm{TRO}}$ Approximation

\begin{tabular}{lcccc}
\hline \hline Data & Type & $N_{l}$ & $N_{u}$ & $S$ \\
\hline S & E2 & 1 & 2 & $5.54 \mathrm{E}-03$ \\
S & M1 & 1 & 2 & $1.77 \mathrm{E}-05$ \\
S & E2 & 1 & 3 & $1.29 \mathrm{E}-02$ \\
S & M1 & 1 & 3 & $6.30 \mathrm{E}-07$ \\
S & E2 & 1 & 4 & $2.25 \mathrm{E}-06$ \\
S & M1 & 1 & 4 & $3.37 \mathrm{E}-04$ \\
S & E2 & 1 & 5 & $3.00 \mathrm{E}-10$ \\
S & M1 & 1 & 5 & $1.67 \mathrm{E}-03$ \\
S & E1 & 1 & 6 & $2.65 \mathrm{E}-01$ \\
S & E1 & 1 & 7 & $1.74 \mathrm{E}-01$ \\
S & M1 & 2 & 3 & $2.40 \mathrm{E}+00$ \\
S & E2 & 2 & 4 & $2.18 \mathrm{E}+01$ \\
S & M1 & 2 & 4 & $2.63 \mathrm{E}-03$ \\
S & E2 & 2 & 5 & $2.17 \mathrm{E}+01$ \\
S & M1 & 2 & 5 & $8.41 \mathrm{E}-03$ \\
\hline
\end{tabular}

Notes. The first column describes the transition data type ( $S$ stands for line strengths $S, \mathrm{~A}$ - for transition rates $A$ ). The second columns describes line type, $N_{l}$ is for the lower level index, and $N_{u}$ denotes the upper level index.

(This table is available in its entirety in a machine-readable form in the online journal. A portion is shown here for guidance regarding its form and content.)

\section{CLOUDY CALCULATIONS}

We have converted the new data to the sTout format, the database introduced in version 13 of Cloudy, the spectral synthesis code last described by Ferland et al. (2013). As described by M. L. Lykins et al. (2013, in preparation; Lykins et al. 2013) and Ferland et al. (2013), Cloudy obtains much of its atomic and molecular data from external files, making it far easier to update and modify the data. For S II, we combine our new calculations of the transition rates with NIST energy levels and collision strengths given by Tayal \& Zatsarinny (2010). We save line strengths $S$ rather than $A_{u l}$ or $g f$ because we use experimental energies $-S$, unlike the other two, does not depend directly on transition energy.

Table 3 lists a few of the calculated transition line strengths $S$; the majority are available in the online journal. The level 


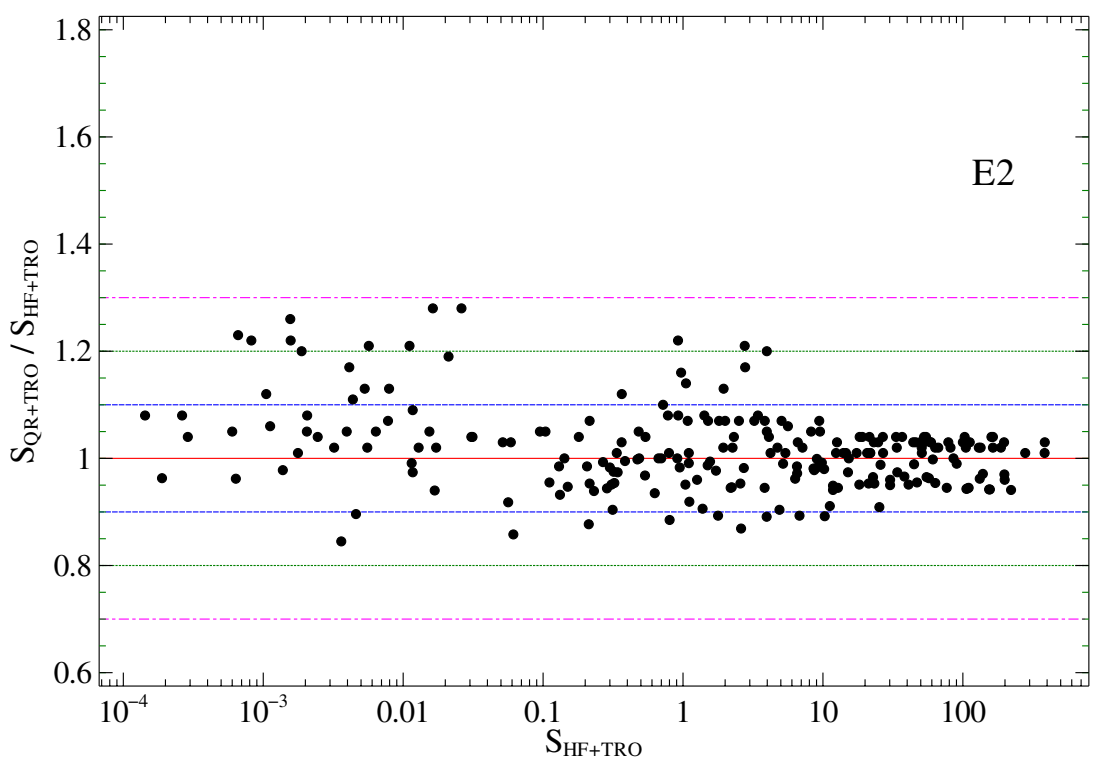

Figure 4. Comparison of the $\mathrm{E} 2$ transition line strengths $S$ determined in the $\mathrm{CI}_{\mathrm{HF}+\mathrm{TRO}}$ and $\mathrm{CI}_{\mathrm{QR}+\mathrm{TRO}}$ approaches for $\mathrm{S}$ II.

(A color version of this figure is available in the online journal.)

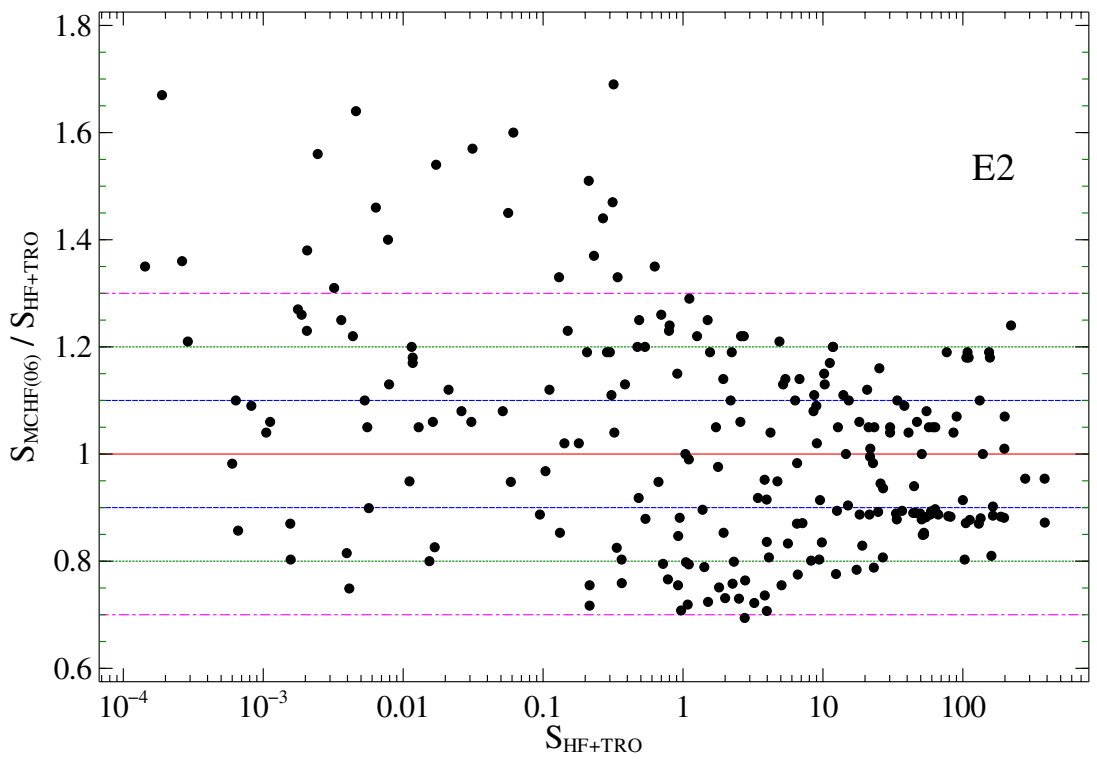

Figure 5. Comparison of the $\mathrm{E} 2$ transition line strengths $S$ determined in the $\mathrm{CI}_{\mathrm{HF}+\mathrm{TRO}}$ and $\mathrm{MCHF}_{06}$ (Froese Fischer et al. 2006) approaches for $\mathrm{S}$ II.

(A color version of this figure is available in the online journal.)

indices are from Table 1, and the experimental level energies given there can be used to derive line wavelengths. These data were produced in the $\mathrm{CI}_{\mathrm{HF}+\mathrm{TRO}}$ approximation. Based on the listed data, one can easily transform transition line strengths $S$ into the $g f$ values or the transition rates $A$ by using available experimental or calculated transition energies. Further details are given in M. L. Lykins et al. (2013, in preparation).

The following sections show representative sulphur spectra and discuss an application to DLAs.

\subsection{Pure- $S^{+}$Emission Spectra}

M. L. Lykins et al. (2013, in preparation) describe our calculation of gas in collisional ionization equilibrium. We show two representative spectra, absorption and emission spectra, in this section.
Two emission spectra of a pure-S gas in coronal equilibrium at $T=2 \times 10^{4} \mathrm{~K}$, the temperature where the fraction of $\mathrm{S}^{+}$ peaks, are shown in Figure 8. Both simulations have a unit volume $\left(1 \mathrm{~cm}^{-3}\right)$ of gas but have different densities, $1 \mathrm{~cm}^{-3}$ and $10^{10} \mathrm{~cm}^{-3}$. The lower density is in the low-density limit and the spectrum would be characteristic of any gas with density $n_{e} \lesssim 10^{3} \mathrm{~cm}^{-3}$. As expected, the denser gas is $\sim 10^{20}$ times more emissive. The higher density is characteristic of quasar emission-line regions (Osterbrock \& Ferland 2006). The prominent feature at $1197 \AA$ is the $\mathrm{S}^{+} \rightarrow \mathrm{S}^{0}$ radiative recombination continuum. Many hundreds of $\mathrm{S}$ II lines are present, providing valuable diagnostics of the gas conditions.

The strongest lines in the lower density (see the upper panel of Figure 8) are the optical forbidden [S II] $\lambda \lambda 6730.82,6716.44$ doublet, a density indicator in nebulae (Osterbrock \& Ferland 2006). The next strongest lines are the optical [S II] 


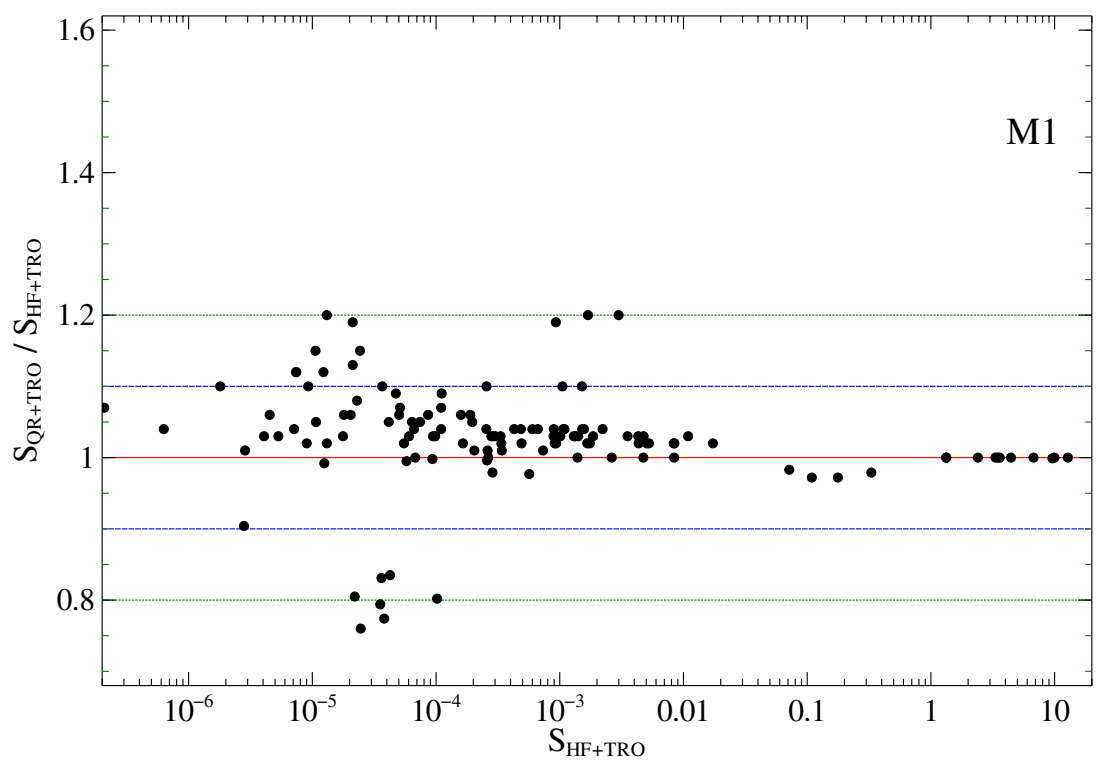

Figure 6. Comparison of the $\mathrm{M} 1$ transition line strengths $S$ determined in the $\mathrm{CI}_{\mathrm{HF}+\mathrm{TRO}}$ and $\mathrm{MCHF}_{06}$ (Froese Fischer et al. 2006) approaches for $\mathrm{S}$ II.

(A color version of this figure is available in the online journal.)

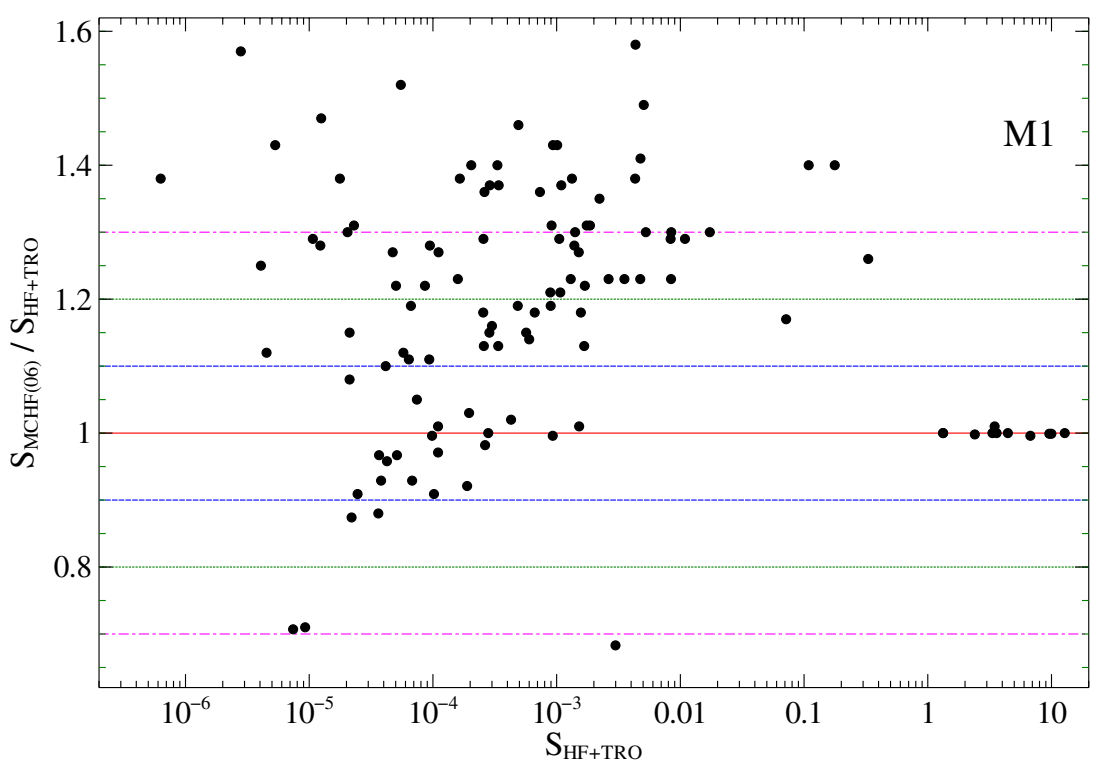

Figure 7. Comparison of the M1 transition line strengths $S$ determined in $\mathrm{CI}_{\mathrm{HF}+\mathrm{TRO}}$ and $\mathrm{MCHF}_{06}$ (Froese Fischer et al. 2006) approaches for $\mathrm{S}$ II. Froese Fischer et al. (2006) approaches for S II.

(A color version of this figure is available in the online journal.)

$\lambda \lambda 4068.60,4076.35$ doublet, which, combined with the previous pair, are a temperature indicator. Multiplets in the NIR at $\lambda \lambda 10320,10336,10287,10370$, and in the FUV at $\lambda \lambda 1259.52,1253.81,1250.58$, are also strong.

The optical and NIR forbidden lines are collisionally suppressed in the denser gas shown in the lower panel. The strongest lines in this case are in the FUV, at the $\lambda \lambda 1259.52,1253.81,1250.58$ multiplet followed by $\lambda \lambda 1204.32,1204.27$. These lines are allowed and are optically thick if the $\mathrm{S}^{+}$column density is large enough.

\subsection{Pure- $S^{+}$Absorption Spectra}

S II FUV lines are commonly observed in absorption in the interstellar and intergalactic media, and can be used to probe the composition of the intervening clouds. The lower panel of Figure 9 shows the absorption spectrum of a pure- $\mathrm{S}^{+}$gas with a column density of $N\left(\mathrm{~S}^{+}\right)=10^{15.2} \mathrm{~cm}^{-2}$. We consider models of DLA clouds in the following section, but present the absorption spectrum of a pure- $\mathrm{S}^{+}$gas here for completeness. The $N\left(\mathrm{~S}^{+}\right)$ column density was chosen to be representative of the column density through the low-metallicity DLA clouds described in the next section. The figure is limited to wavelengths $\lambda>1000 \AA$ since shorter regions are likely to be blocked by Lyman-limit confusion.

Only a few $\mathrm{S}$ II lines (three lines near $1250 \AA$ ) are present in the spectral region shown in Figure 9. Other strong S II absorption lines are present, however. Table 4 lists all predicted $\mathrm{S}_{\text {II }}$ lines with optical depths greater than $10^{-3}$. Most of the lines are in the EUV, making them unobservable at cosmological distances; however, we note that there are two multiplets near $1021 \AA$ and $1204 \AA$ that would be observable in higher column density objects. 


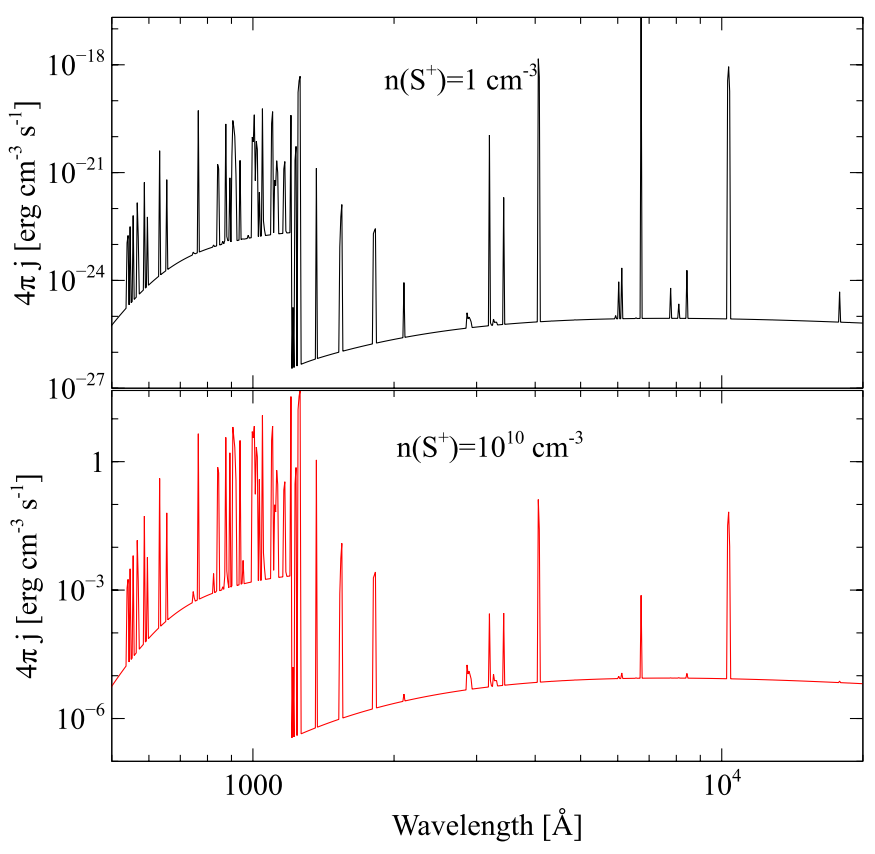

Figure 8. S II emission spectra of a unit cell of pure- $\mathrm{S}^{+}$gas in coronal equilibrium at a temperature of $2 \times 10^{4} \mathrm{~K}$. Two $\mathrm{S}^{+}$densities, $1 \mathrm{~cm}^{-3}$ and $10^{10} \mathrm{~cm}^{-3}$, are shown. The low density produces a spectrum that peaks in the optical/NIR while the high density case emits mainly in the FUV.

(A color version of this figure is available in the online journal.)

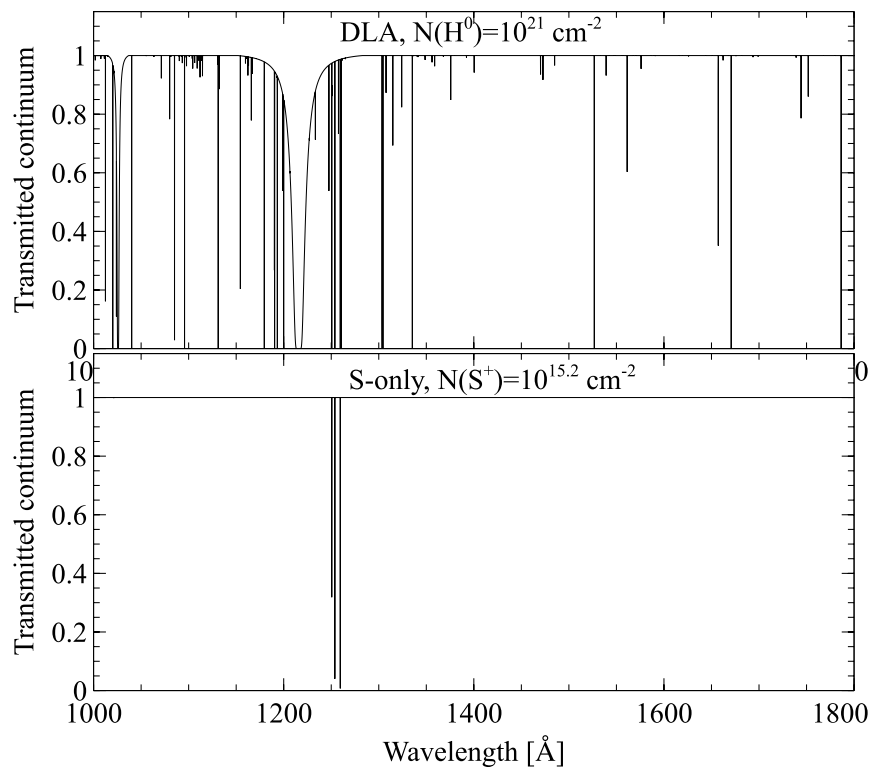

Figure 9. S II absorption plot. The upper panel shows a full DLA spectrum, with absorption lines of various elements shown relative to a normalized continuum. The strongest line is the damped Ly $\alpha$ line. The lower panel shows a spectrum in which all $\mathrm{S}$ is singly ionized. The $\mathrm{S}^{+}$column density through the cell in the lower panel is similar to the $\mathrm{S}^{+}$column density in the DLA shown in the upper panel.

\subsection{Application to DLAs}

One aim of DLA absorption-line spectroscopy is to be able to measure elemental abundances. In the case of S, we are often limited to the $\mathrm{S}$ II lines described in this paper. These can be used to infer the $\mathrm{S}^{+}$column density, but to get abundances we must estimate the ionization fraction ratio $\mathrm{S}^{+} / \mathrm{S}$.

Howk \& Sembach (1999) pointed out that certain ion ratios can be used to estimate the ionization fractions of elements
Table 4

$\mathrm{S}$ II Mean Optical Depths for the $\mathrm{S}^{+}$-absorbing Cloud in Figure 9

\begin{tabular}{|c|c|c|}
\hline Ion & $\begin{array}{c}\lambda \\
(\AA)\end{array}$ & Mean Optical Depth \\
\hline $\mathrm{S}_{\text {II }}$ & 538 & $1.18 \mathrm{E}+00$ \\
\hline $\mathrm{S}_{\text {II }}$ & 538 & $9.32 \mathrm{E}+00$ \\
\hline S II & 541 & $1.39 \mathrm{E}+01$ \\
\hline S II & 542 & $1.71 \mathrm{E}+00$ \\
\hline $\mathrm{S}_{\text {II }}$ & 546 & $2.22 \mathrm{E}+01$ \\
\hline$S_{\text {II }}$ & 547 & $2.61 E+00$ \\
\hline $\mathrm{S}_{\text {II }}$ & 554 & $3.86 \mathrm{E}+01$ \\
\hline $\mathrm{S}_{\text {II }}$ & 555 & $4.29 \mathrm{E}+00$ \\
\hline$S_{\text {II }}$ & 566 & $7.60 \mathrm{E}+01$ \\
\hline $\mathrm{S}_{\text {II }}$ & 569 & $7.81 \mathrm{E}+00$ \\
\hline $\mathrm{S}_{\text {II }}$ & 587 & $1.81 \mathrm{E}+02$ \\
\hline $\mathrm{S}_{\text {II }}$ & 595 & $1.71 \mathrm{E}+01$ \\
\hline S II & 632 & $6.12 \mathrm{E}+02$ \\
\hline $\mathrm{S}_{\text {II }}$ & 654 & $6.77 \mathrm{E}+01$ \\
\hline $\mathrm{S}_{\text {II }}$ & 750 & $3.27 \mathrm{E}+03$ \\
\hline $\mathrm{S}_{\text {II }}$ & 888 & $6.25 \mathrm{E}+02$ \\
\hline $\mathrm{S}_{\text {II }}$ & 910 & $2.35 \mathrm{E}+02$ \\
\hline $\mathrm{S}_{\text {II }}$ & 912 & $1.17 \mathrm{E}+02$ \\
\hline $\mathrm{S}_{\text {II }}$ & 1021 & $1.50 \mathrm{E}-02$ \\
\hline S II & 1021 & $1.56 \mathrm{E}-03$ \\
\hline $\mathrm{S}_{\text {II }}$ & 1204 & $1.34 \mathrm{E}-03$ \\
\hline S II & 1250 & $1.21 \mathrm{E}+01$ \\
\hline $\mathrm{S}_{\text {II }}$ & 1253 & $2.44 \mathrm{E}+01$ \\
\hline$S_{\text {II }}$ & 1259 & $3.72 \mathrm{E}+01$ \\
\hline
\end{tabular}

where only one stage of ionization is seen. We redo a calculation in the spirit of theirs. Like Howk \& Sembach (1999), the spectral energy distribution (SED) is from Haardt \& Madau (1996) and the total neutral hydrogen column density of the cloud is taken as $N\left(\mathrm{H}^{0}\right)=10^{21} \mathrm{~cm}^{-2}$, roughly in the middle of the range of DLAs. We assume a redshift of $z=2$. We assume ISM gas-phase abundances and dust with the metallicity and dust-to-gas ratio reduced by $1 \mathrm{dex}$, as is typical of these objects.

Given these assumptions, the only free parameter is the gas density. The metagalactic radiation background is assumed to be the only source of ionization. Given this SED, the ionization of a DLA will be determined by its density, since the impinging flux of photons is constant. In this case, lower density gas will have a high ionization parameter (Osterbrock \& Ferland 2006). The density range was chosen to cover the range of ionization parameters shown in Howk \& Sembach (1999).

Figure 10 shows the results. The upper panel gives some observable ion ratios, while the lower panel shows the computed $\mathrm{S}^{+}$ ionization fraction. Clouds with densities greater than $\sim 1 \mathrm{~cm}^{-3}$ will have nearly all $\mathrm{S}$ in the form of $\mathrm{S}^{+}$.

The full DLA spectrum of a cloud with a hydrogen density of $1 \mathrm{~cm}^{-3}$, so that $\mathrm{S}^{+}$is the dominant ion stage and $N\left(\mathrm{H}^{0}\right)=$ $10^{21} \mathrm{~cm}^{-2}$, is shown in the upper panel of Figure 9. The $\mathrm{S}^{+}$ column density in the lower panel was chosen to be similar to the $\mathrm{S}^{+}$column density through the DLA shown in the upper panel. These figures illustrate the potential of the new $\mathrm{S}$ II data for the purpose of making comparisons to observed spectra.

\section{DISCUSSION}

Our oscillator strengths for most S II absorption lines agree closely with previous calculations. For example, for the triplet at $1250.6,1253.8$, and $1259.5 \AA$, our calculations are lower than 


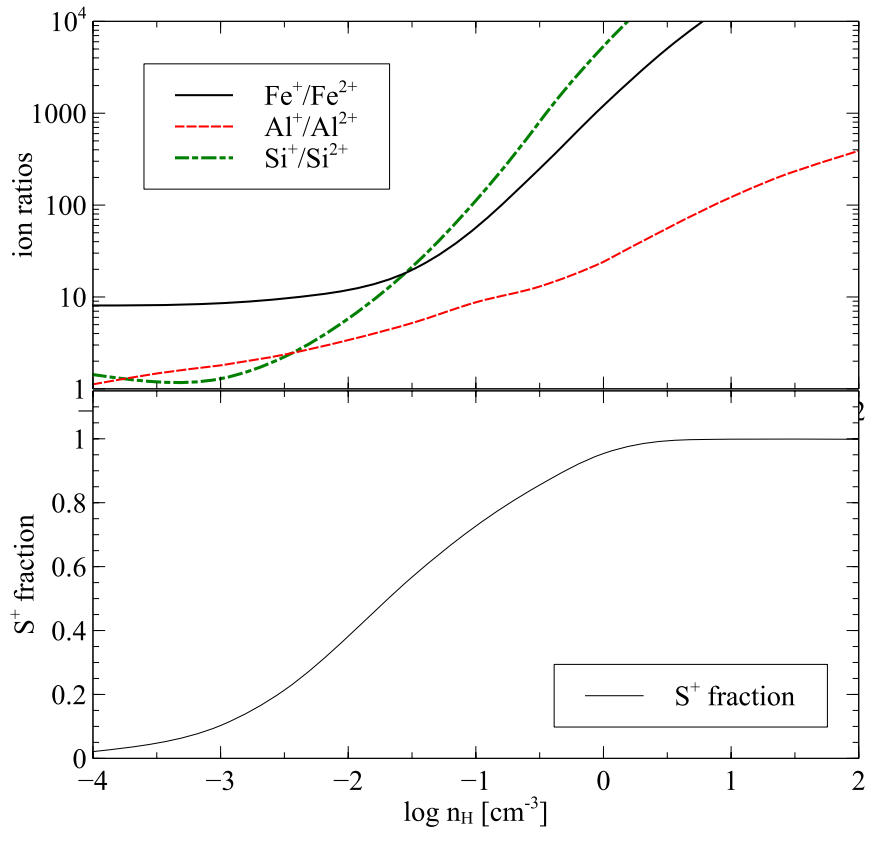

Figure 10. Upper panel: ratios of several commonly observed ions. Lower panel: predicted $\mathrm{S}^{+}$ionization fraction. Several ratios correlate with $\mathrm{S}^{+}$and can be used to estimate it.

(A color version of this figure is available in the online journal.)

previous ones by 0.04 dex. Thus, the metallicities inferred from these lines would be higher by 0.04 dex. It is reassuring that these metallicity corrections are relatively small.

One surprising result of our work is that the uncertainties NIST placed on the existing $\mathrm{S}_{\text {II }}$ transition rates were too pessimistic. Their quality flags indicate a typical uncertainty of roughly $30 \%$. Our independent calculations confirm the predictions of previous work, and suggest that the typical uncertainty is closer to $10 \%$ for the strongest lines.

Our Cloudy simulations illustrate an astrophysical application of the atomic data calculations, and the resultant predictions for a large number of emission and absorption lines. Such predictions can be compared with observed line strengths to better constrain the properties of distant galaxies. Past S II absorption-line observations of DLAs usually focused on the strong lines at $\lambda \lambda 1250.58,1253.81,1259.52$. Our calculations confirm that these are the strongest lines in the observable part of the spectrum. Our full S II dataset, which is posted online, contains many UV transitions, some of which could be detected in high-signal-to-noise spectra with future large telescopes.

It is also important to estimate how much difference from the true $[\mathrm{S} / \mathrm{H}]$ can be caused by ionization effects. Our Cloudy calculations in Figure 10 indicate that the ionization correction for $[\mathrm{S} / \mathrm{H}]$ derived from $\mathrm{S}_{\mathrm{II}} / \mathrm{H}_{\mathrm{I}}$ is small for typical DLAs. We note, however, that these calculations are subject to uncertainties in recombination coefficients. We plan to perform improved calculations of recombination coefficients in another part of this study.

We also note that theoretically calculated transition wavelengths can never reach the accuracy of experimental data. As is the standard practice in this field, we use experimental $\lambda$ values in our Cloudy simulation runs. Transition probabilities or oscillator strengths must be corrected for the difference between experimental and theoretical energies, which is why our calculations work in terms of the line strength $S$. We correct our transition rates by introducing the experimental level energies to determine radiative transition parameters, such as oscillator strengths $g f$, transition probabilities $A$, or tabulated data.

This paper is a demonstration of the work we plan to do for other ions of $\mathrm{S}$ as well as for the observationally important ions of other elements from $\mathrm{Al}$ to $\mathrm{Zn}$. The results of these broader calculations will be presented in several future papers and made available to the astrophysics community through incorporation into Cloudy.

This work is supported by the National Science Foundation grant AST/1109061 to the University of Kentucky and AST/ 1108830 to the University of South Carolina. V.P.K. also acknowledges partial support from the NSF (AST/0908890) and NASA (HST-GO-12536). G.J.F. acknowledges additional support by the NSF (AST/1108928), NASA (10-ATP10-0053, 10-ADAP10-0073, and NNX12AH73G), and STScI (HST-AR12125.01, HST-AR- 13245, GO-12560, and HST-GO-12309). R.K.'s and P.B.'s research is partially funded the European Social Fund under the Global Grant measure, project VP1-3.1-ŠMM07-K-02-013.

\section{REFERENCES}

Battisti, A. J., Meiring, J. D., Tripp, T. M., et al. 2012, ApJ, 744, 93

Bogdanovich, P. 2004, LitJP, 44, 135

Bogdanovich, P. 2005, NIMPB, 235, 92

Bogdanovich, P., \& Karpuškienè, R. 2001, CoPhC, 134, 321

Bogdanovich, P., Karpuškienè, R., \& Momkauskaitė, A. 2002, CoPhC, 143,174

Bogdanovich, P., Karpuškienè, R., \& Martinson, I. 2003, PhyS, 67, 44

Bogdanovich, P., \& Kisielius, R. 2012, ADNDT, 98, 557

Bogdanovich, P., \& Kisielius, R. 2013, ADNDT, 99, 580

Bogdanovich, P., \& Rancova, O. 2006, PhRvA, 74, 052501

Bogdanovich, P., \& Rancova, O. 2007, PhRvA, 76, 012507

Bogdanovich, P., \& Rancova, O. 2008, PhyS, 78, 045301

Chen, H.-W., Prochaska, J. X., Bloom, J. S., \& Thompson, I. B. 2005, ApJL, 634, L25

Cooke, R., Pettini, M., Steidel, C. C., Rudie, G. C., \& Nissen, P. E. 2011, MNRAS, 417, 1534

Cowan, R. D. 1981, in The Theory of Atomic Structure and Spectra (Los Angeles, LA: Univ. California Press)

Ferland, G. J., Porter, R. L., van Hoof, P. A. M., et al. 2013, RMxAA, 49, 1

Fritzsche, S., Fricke, B., Geschke, D., Heitman, A., \& Sienkiewicz, J. E. 1999, ApJ, 518, 994

Froese Fischer, C., Tachiev, G., \& Irimia, A. 2006, ADNDT, 92, 607

Fynbo, J. P. U., Jakobsson, P., Prochaska, J. X., et al. 2009, ApJS, 185, 526

Guimaraes, R., Noterdaeme, P., Petitjean, P., et al. 2012, AJ, 143, 147

Haardt, F., \& Madau, P. 1996, ApJ, 461, 20

Howk, J. C., \& Sembach, K. R. 1999, ApJL, 523, L141

Irimia, A., \& Froese Fischer, C. 2005, PhyS, 71, 172

Jenkins, E. B. 2009, ApJ, 700, 1299

Jonauskas, V., Bogdanovich, P., Keenan, F. P., et al. 2005, A\&A, 433, 745

Jonauskas, V., Bogdanovich, P., Keenan, F. P., et al. 2006, A\&A, 455, 1157

Jorgenson, R. A., Murphy, M. T., \& Thompson, R. 2013, MNRAS, 435, 482

Karpuškienè, R., \& Bogdanovich, P. 2009, ADNDT, 95, 533

Keenan, F. P., Hibbert, A., Ojha, P. C., \& Conlon, E. S. 1993, PhyS, 48, 129

Kramida, A., Ralchenko, Y., Reader, J., \& NIST ASD Team, 2013, in NIST Atomic Spectra Database (version 5.1), (Gaithersburg, MD: National Institute of Standards and Technology)

Kulkarni, V. P., \& Fall, S. M. 2002, ApJ, 580, 732

Kulkarni, V. P., Khare, P., Péroux, C., et al. 2007, ApJ, 661, 88

Kulkarni, V. P., Meiring, J., Som, D., et al. 2012, ApJ, 749, 176

Lykins, M. L., Ferland, G. J., Porter, R. L., et al. 2013, MNRAS, 429, 3133

Meiring, J. D., Kulkarni, V. P., Lauroesch, J. T., et al. 2009, MNRAS, 397, 2037

Mendoza, C., \& Zeippen, C. J. 1982, MNRAS, 198, 127

Morton, D. C. 2003, ApJS, 149, 205

Osterbrock, D. E., \& Ferland, G. J. 2006, Astrophysics of Gaseous Nebulae and Active Galactic Nuclei (2nd ed.; Mill Valley, CA: Univ. Science Press), (AGN3) 
Péroux, C., Dessauges-Zavadsky, M., D’Odorico, S., et al. 2005, MNRAS, 363 479

Péroux, C., Meiring, J. D., Kulkarni, V. P., et al. 2008, MNRAS, 386, 2209

Pettini, M., Smith, L. J., King, D. L., \& Hunstead, R. W. 1997, ApJ, 486, 665

Podobedova, L. I., Kelleher, D. E., \& Wiese, W. L. 2009, JPCRD, 38, 171

Prochaska, J. X., Chen, H.-W., Dessauges-Zavadsky, M., et al. 2007, ApJ, 666,267

Prochaska, J. X., Gawiser, E., Wolfe, A. M., Castro, S., \& Djorgovski, S. G. 2003, ApJL, 595, L9
Prochaska, J. X., Herbert-Fort, S., \& Wolfe, A. M. 2005, ApJ, 635, 123

Rafelski, M., Wolfe, A. M., Prochaska, J. X., Neeleman, M., \& Mendez, A. J. 2012, ApJ, 755, 89

Savage, B. D., \& Sembach, K. R. 1996, ARA\&A, 34, 279

Savaglio, S., Fall, S. M., \& Fiore, F. 2003, ApJ, 585, 638

Som, D., Kulkarni, V. P., Meiring, J., et al. 2013, MNRAS, 435, 1469

Storrie-Lombardi, L. J., \& Wolfe, A. M. 2000, ApJ, 543, 552

Tayal, S. S., \& Zatsarinny, O. 2010, ApJSS, 188, 32

Verner, D. A., Verner, E. M., \& Ferland, G. J. 1996, ADNDT, 64, 1 